\title{
More on Spectral Analysis of Signed Networks
}

\author{
Guihai Yu (D) ${ }^{1}$ and Hui Qu $\mathbf{u}^{1,2}$ \\ ${ }^{1}$ School of Mathematics and Statistics, Guizhou University of Finance and Economics, Guiyang, Guizhou, 550025, China \\ ${ }^{2}$ Shandong Co-Innovation Center of Future Intelligent Computing, Yantai, Shandong, 264005, China
}

Correspondence should be addressed to Guihai Yu; yuguihai@126.com

Received 9 August 2018; Accepted 27 September 2018; Published 16 October 2018

Guest Editor: Piotr Brodka

\begin{abstract}
Copyright ( 2018 Guihai Yu and Hui Qu. This is an open access article distributed under the Creative Commons Attribution License, which permits unrestricted use, distribution, and reproduction in any medium, provided the original work is properly cited.

Spectral graph theory plays a key role in analyzing the structure of social (signed) networks. In this paper we continue to study some properties of (normalized) Laplacian matrix of signed networks. Sufficient and necessary conditions for the singularity of Laplacian matrix are given. We determine the correspondence between the balance of signed network and the singularity of its Laplacian matrix. An expression of the determinant of Laplacian matrix is present. The symmetry about 1 of eigenvalues of normalized Laplacian matrix is discussed. We determine that the integer 2 is an eigenvalue of normalized Laplacian matrix if and only if the signed network is balanced and bipartite. Finally an expression of the coefficient of normalized Laplacian characteristic polynomial is present.
\end{abstract}

\section{Introduction}

Social networks represent a large proportion of the complex socioeconomic organization in modern society which represent social entities including countries, corporations, or people. These entities interconnected through a wide range of social ties such as political treaties, commercial trade, friend, and collaboration. To display the ally/enemy, friend/foe, and trust/distrust relationships, the social system can be well represented by a signed network in which an edge of the network is assigned to be positive if two individuals are ally, friendship, trust, and negative if they are enemy, foe, and distrust. The origin of the study of signed networks can be tracked back to the work of Heider [1]. The use of signed networks was then proposed by Cartwright and Harary [2] to model the existence of balance/unbalance in the social networks.

As we know, graphs are very useful ways of presenting information about signed networks. However, when there are many actors and/or many kinds of relations, they can become so visually complicated that it is very difficult to see patterns. It is also possible to present information about signed networks in the form of matrices. Representing the information in this way also allows the application of mathematical and computer tools to summarize and find patterns.
Up to now, some matrices are employed by signed networks analysts in a number of different ways. This is the so-called spectral graph theory, which is a branch of mathematical science. Its idea is to exploit numerous relationship between the structure of a network (graph) and the spectrum of some matrix (or collection of matrices) associated with the network (graph). There are many different matrices that are employed, including adjacency matrix, Laplacian matrix, and normalized Laplacian matrix. The goal of this paper is to investigate some properties of Laplacian matrix and normalized Laplacian matrix of signed networks and exploit some relation between these matrices and signed networks.

Let $G$ be an undirected network of order $n$ with vertex set $V(G)=\left\{v_{1}, v_{2}, \ldots, v_{n}\right\}$ and edge set $E(G)$. The adjacency matrix $A(G)=\left(a_{i j}\right)_{n \times n}$ of $G$ is defined as follows: $a_{i j}=1$ if $v_{i}$ and $v_{j}$ are adjacent and $a_{i j}=0$ otherwise. A signed network $\Gamma=(G, \sigma)$ consists of a network $G=(V, E)$, referred to as its underlying network, and a sign function $\sigma: E \longrightarrow\{+,-\}$. The adjacency matrix of $\Gamma$ is $A(\Gamma)=$ $\left(a_{i j}^{\sigma}\right)$ with $a_{i j}^{\sigma}=\sigma\left(v_{i} v_{j}\right) a_{i j}$, where $a_{i j}$ is an element in the adjacency matrix of the underlying network $G$ and $v_{i} v_{j}$ is an edge of $G$. If all edges are signed positive, the adjacency matrix $A(G, \sigma)$ is exactly the ordinary adjacency matrix $A(G)$. Let $D(\Gamma)=\operatorname{diag}\left(d_{1}, d_{2}, \ldots, d_{n}\right)$ be a diagonal matrix where 
$d_{i}$ is the degree of vertex $v_{i}$ in its underlying network. The Laplacian matrix of $\Gamma$, denoted by $L(\Gamma)$, is defined as $D(\Gamma)-$ $A(\Gamma)$. The matrix $D^{-1 / 2} L(\Gamma) D^{-1 / 2}$ is said to be normalized Laplacian matrix of $\Gamma$, denoted by $\mathscr{L}(\Gamma)$.

A signed $i_{1}-i_{k}$-walk $W$ in a signed network $\Gamma$ is a sequence of vertices and edges $W: v_{i_{1}} e_{12} v_{i_{2}} e_{23} v_{i_{3}} \cdots v_{i_{k-1}} e_{(k-1) k} v_{i_{k}}$ such that $e_{s(s+1)}=v_{i_{s}} v_{i_{s+1}} \in E(\Gamma)(s=1,2, \ldots, k-1)$. An $i_{1}-i_{k^{-}}$ walk $W$ is called even (odd) if $k$ is even (odd). The sign of a signed walk $W=v_{1} e_{12} v_{2} e_{23} \cdots v_{l}$ is $\operatorname{sgn}(W)=a_{12}^{\sigma} a_{23}^{\sigma} \cdots a_{(l-1) l}^{\sigma}$ and $e_{i(i+1)}=v_{i} v_{i+1}(i=1,2, \ldots, l-1)$. A signed walk $W$ is balanced (unbalanced) if $\operatorname{sgn}(W)=1(\operatorname{sgn}(W)=-1)$. A signed cycle is called balanced (unbalanced) if its sign is +1 $(-1)$. A signed networks is called balanced (resp. unbalanced) if each its signed cycle is balanced (resp. unbalanced).

Suppose that $\Gamma=(G, \sigma)$ is a signed network. A signed function $\theta: V \longrightarrow\{+1,-1\}$ is a switching function if $\Gamma$ is transformed to a new signed network $\Gamma^{\theta}=\left(G, \sigma^{\theta}\right)$ by $\theta$ such that the underlying graph remains the same and the sign function is defined by $\sigma^{\theta}(e)=\theta\left(v_{i}\right) \sigma(e) \theta\left(v_{j}\right)$ for an edge $e=v_{i} v_{j}$. Let $\Gamma_{1}=\left(G, \sigma_{1}\right)$ and $\Gamma_{2}=\left(G, \sigma_{2}\right)$ be two signed networks with the same underlying graph. We call $\Gamma_{1}$ and $\Gamma_{2}$ switching equivalent and write $\Gamma_{1} \sim \Gamma_{2}$, if there exists a switching function $\theta$ such that $\Gamma_{2}=\Gamma_{1}^{\theta}$. Switching preserves some signed-graphic invariants such as the sign of cycles and spectrum of combinatorial matrices (adjacency matrix, normalized Laplacian matrix).

This paper is organized as follows. In Section 2, we study some properties of Laplacian matrix of signed networks. Sufficient and necessary conditions for the singularity of Laplacian matrix are given. The correspondence between the balance of signed network and the singularity of its Laplacian matrix is determined. An expression of the determinant of Laplacian matrix is present. In Section 3, the symmetry about 1 of eigenvalues of normalized Laplacian matrix is discussed. Sufficient and necessary condition for that the integer 2 is an eigenvalue of normalized Laplacian matrix is given. An expression of all coefficients of normalized Laplacian characteristic polynomial is present.

\section{Laplacian Matrix and Signed Network}

Hou et al. [3] introduced the incidence matrix of a signed network as follows. Let $S(\Gamma)=\left(s_{i j}\right)$ be an $n \times m$ matrix indexed by the vertex and the edge of signed network $\Gamma$ and

$$
s_{i j}= \begin{cases}+1 & \text { if } v_{i} \text { is the head of } e_{j} \\ +1 & \text { if } v_{i} \text { is the tail of } e_{j} \text { and } \sigma\left(e_{j}\right)=+ \\ -1 & \text { if } v_{i} \text { is the tail of } e_{j} \text { and } \sigma\left(e_{j}\right)=- \\ 0 & \text { otherwise. }\end{cases}
$$

The following is immediate by the direct calculation.

Theorem 1 (see [3]). Let $\Gamma$ be a signed network. Then $L(\Gamma)=$ $S(\Gamma) S^{T}(\Gamma)$ and $L(\Gamma)$ is a positive semidefinite matrix.

Theorem 2. Let $\Gamma$ be a connected signed network on vertices $v_{1}, v_{2}, \ldots, v_{n}$. Then $L(\Gamma)$ is singular if and only if any $1-i$-walk has the same sign. In this case, 0 is a simple eigenvalue with an eigenvector $\alpha=\left(1, \operatorname{sgn}\left(W_{2}\right), \operatorname{sgn}\left(W_{3}\right), \ldots, \operatorname{sgn}\left(W_{n}\right)\right)^{T}$, where $W_{i}$ is a $1-i$-walk in $\Gamma$.

Proof. Let $x^{T}=\left(x_{1}, x_{2}, \ldots, x_{n}\right) \in C^{n}$. Note that for any nonzero vector $x, L(\Gamma)=0$ if and only if $S^{T}(\Gamma) x=0$. By (14), $S^{T}(\Gamma) x=0$ if and only if $x_{i}=a_{i j}^{\sigma} x_{j}$ for any edge $e=v_{i} v_{j}$. Let $W_{i}=u_{1} u_{2} \cdots u_{i}$ be any $1-i$-walk and $u_{1}=v_{1}, u_{i}=v_{i}$. Suppose that $L(\Gamma)=0$. So we have

$$
\begin{aligned}
x_{1} & =a_{12}^{\sigma} x_{2}=a_{12}^{\sigma} a_{23}^{\sigma} x_{3}=\cdots=a_{12}^{\sigma} a_{23}^{\sigma} \cdots a_{(i-1) i}^{\sigma} x_{i} \\
& =\operatorname{sgn}\left(W_{i}\right) x_{i},
\end{aligned}
$$

which implies that each $1-i$-walk has the same sign.

Note that $\operatorname{sgn}^{-1}\left(W_{i}\right)=\operatorname{sgn}\left(W_{i}\right)$. Hence

$$
\begin{aligned}
x^{T} & =\left(x_{1}, x_{2}, \ldots, x_{n}\right) \\
& =\left(x_{1}, \operatorname{sgn}\left(W_{2}\right) x_{1}, \operatorname{sgn}\left(W_{3}\right) x_{1}, \ldots, \operatorname{sgn}\left(W_{n}\right) x_{1}\right) \\
& =x_{1} \alpha^{T} .
\end{aligned}
$$

This implies that 0 is a simple eigenvalue of $L(\Gamma)$ with an eigenvector $\alpha$.

Suppose that any $1-i$-walk has the sign. Then for any edge $e_{i j} \in E(\Gamma), \operatorname{sgn}\left(W_{j}\right)=\operatorname{sgn}\left(W_{i}\right) \cdot a_{i j}^{\sigma}$. Let $x^{T}=\left(x_{1}, x_{2}, \ldots, x_{n}\right)$ be a column vector such that $x_{i}=\operatorname{sgn}\left(W_{i}\right) x_{1}(i=2,3, \ldots, n)$. Then $x_{j}=\operatorname{sgn}\left(W_{j}\right) x_{1}=\operatorname{sgn}\left(W_{i}\right) \cdot a_{i j}^{\sigma} x_{1}=x_{i} a_{i j}^{\sigma}$, i.e., $x_{i}=a_{i j}^{\sigma} x_{j}$. So we have

$$
\begin{aligned}
x^{T} L(\Gamma) x & =\sum_{e_{i j} \in E(\Gamma)}\left|x_{i}-a_{i j}^{\sigma} x_{j}\right|^{2}=\sum_{e_{i j} \in E(\Gamma)}\left|a_{i j}^{\sigma} x_{j}-a_{i j}^{\sigma} x_{j}\right|^{2} \\
& =0 .
\end{aligned}
$$

This implies that $L(\Gamma)$ is singular.

Note that for the underlying network it is known that the multiplicity of the eigenvalue 0 of Laplacian matrix is equal to the number of components. For signed network, the following holds from the proof of Theorem 2 .

Theorem 3. The multiplicity of the eigenvalue 0 of Laplacian matrix of a signed network is the number of components whose Laplacian matrix is singular.

Theorem 4 (see [4]). A signed network is balanced if and only if for each pair of distinct vertices $v_{1}, v_{2}$ all paths joining $v_{1}$ and $v_{2}$ have the same sign.

From Theorems 2 and 4, we have the following.

Theorem 5. A signed network $\Gamma$ is balanced if and only if $L(\Gamma)$ is singular.

The following is immediate from Theorem 5.

Theorem 6. The Laplacian matrix of a signed network is singular if and only if the Laplacian matrix of any its cycles is singular. In particular, the Laplacian matrix of any acyclic graph is singular. 
In [5], authors determined the determinant of the Laplacian matrix of mixed graphs. Here by the similar method we shall extend it to the case for signed graphs.

Theorem 7. $\operatorname{det} L(C)=2[1-\operatorname{sgn}(C)]$ for any signed cycle $C$.

Proof. Let $C$ be a signed cycle with vertex set $V(C)=$ $\left\{v_{1}, v_{2}, \ldots, v_{n}\right\}$ and edge set $E(C)=\left\{e_{1}, e_{2}, \ldots, e_{n}\right\}$ such that $e_{i}=v_{i} v_{i+1}(1 \leq i \leq n-1)$ and $e_{n}=v_{n} v_{1}$. For the incidence matrix $S(C)$, we expand its the first row

$$
\operatorname{det} S(C)=\prod_{i=1}^{n} s_{i e_{i}}+(-1)^{n+1} s_{1 e_{n}} \prod_{i=2}^{n} s_{i e_{i-1}} .
$$

By directly calculation and the fact that $s_{i e_{i}} s_{j e_{j}}=-\sigma(e) a_{i j}=$ $-a_{i j}^{\sigma}$ for any edge $e_{\mathrm{i}}=v_{i} v_{i+1}$. It follows that

$$
\operatorname{det} L(C)=\operatorname{det} S(C) \cdot \operatorname{det} S^{T}(C)=2-2 \operatorname{sgn}(C) .
$$

So the result holds.

Theorem 8. Let $\Gamma$ be a signed unicyclic network with a cycle C. Then

$$
\operatorname{det} L(\Gamma)=\operatorname{det} L(C)=2[1-\operatorname{sgn}(C)] .
$$

Proof. By Theorem 7, the results hold if $\Gamma$ is a signed cycle. Assume that $\Gamma$ has a pendant vertex, say $u$. Let $v$ be the unique neighbor of $u$ in $\Gamma$. Let $e$ be the edge joining $u$ and $v$. After permutations, the first row and the first column of $S(\Gamma)$ correspond to the vertex $u$ and the edge $e$, respectively. Note that $S(\Gamma)$ is a square matrix since $\Gamma$ is unicyclic. We get the determinant of $S(\Gamma)$ by expanding along the first row as follows:

$$
\operatorname{det} S(\Gamma)=s_{u e} \cdot \operatorname{det} S\left(\Gamma^{\prime}\right)
$$

where $\Gamma^{\prime}$ is a signed subgraph obtained from $\Gamma$ by deleting the vertex $u$. Hence we have

$$
\begin{aligned}
\operatorname{det} L(\Gamma) & =\operatorname{det} S(\Gamma) \cdot \operatorname{det} S^{T}(\Gamma) \\
& =\operatorname{det} S\left(\Gamma^{\prime}\right) \cdot \operatorname{det} S^{T}\left(\Gamma^{\prime}\right)=\operatorname{det} L\left(\Gamma^{\prime}\right) .
\end{aligned}
$$

Repeating the above finite steps, we have $\operatorname{det} L(\Gamma)=\operatorname{det} L(C)$.

Let $\Gamma$ be a connected signed network. We call a subnetwork $H$ as an essential spanning subnetwork of $\Gamma$ if either $\Gamma$ is balanced and $H$ is a spanning tree of $\Gamma$, or else $\Gamma$ is not balanced, $V(\Gamma)=V(H)$ and every component of $H$ is a unicyclic signed network in which the unique cycle is negative. By $\mathscr{E}(\Gamma)$ we denote the set of all essential spanning subnetworks of $\Gamma$.

Theorem 9. Let $\Gamma$ be a connected signed network. Then

$$
\operatorname{det} L(\Gamma)=\sum_{l=0} 4^{l} b_{l},
$$

where $b_{l}$ is the number of essential spanning subgraphs which contain l unbalanced cycles and $b_{0}=0$.
Proof. It is evident that the result holds if $\Gamma$ is a tree. Assume that $\Gamma$ contains some cycles. By Cauchy-Binet Theorem [6] and $L(\Gamma)=S(\Gamma) \cdot S^{T}(\Gamma)$, we have

$$
\begin{aligned}
\operatorname{det} L(\Gamma)= & \sum_{E^{\prime} \subseteq E(\Gamma) ;\left|E^{\prime}\right|=|V(\Gamma)|} \operatorname{det} S\left[V(\Gamma), E^{\prime}\right] \\
& \cdot \operatorname{det} S^{T}\left[V(\Gamma), E^{\prime}\right],
\end{aligned}
$$

where $S\left[V(\Gamma), E^{\prime}\right]$ is a square submatrix of $S(\Gamma)$.

Note that $S\left[V(\Gamma), E^{\prime}\right]$ is the vertex-edge incidence matrix of a spanning subgraph of $\Gamma$, say $H_{E^{\prime}}$, with the edge set $\left|E^{\prime}\right|=$ $|V(\Gamma)|$. Moreover, $\operatorname{det} L\left(H_{E^{\prime}}\right)=S\left[V(\Gamma), E^{\prime}\right] \cdot S^{T}\left[V(\Gamma), E^{\prime}\right]$. Note that every component of $H_{E^{\prime}}$ is unicyclic and $H_{E^{\prime}} \in$ $\mathscr{E}(\Gamma)$. By Theorem 8 , we have

$$
\begin{aligned}
& \operatorname{det} L(\Gamma)= \sum_{E^{\prime} \subseteq E(\Gamma) ;\left|E^{\prime}\right|=|V(\Gamma)|} \operatorname{det} S\left[V(\Gamma), E^{\prime}\right] \\
& \cdot \operatorname{det} S^{T}\left[V(\Gamma), E^{\prime}\right] \\
&= \sum_{E^{\prime} \subseteq E(\Gamma) ;\left|E^{\prime}\right|=|V(\Gamma)|} \operatorname{det} L\left(H_{E^{\prime}}\right) \\
&= \sum_{H \in \mathscr{E}(\Gamma)} \operatorname{det} L(H) \\
&= \sum_{H \in \mathscr{E}(\Gamma)} \prod_{i=1}^{b_{l}} 2\left(1-\operatorname{sgn}\left(C_{i}(H)\right)\right) \\
& \text { where } \operatorname{sgn}\left(C_{i}(H)\right)=-1=\sum_{l=0} 4^{l} b_{l} .
\end{aligned}
$$

So the result holds.

The following is immediate from Theorem 9, which is coincident with the definition of balance of signed network.

Theorem 10. Let $\Gamma$ be a signed network. Then $\Gamma$ is balanced if and only if each cycle of $\Gamma$ is balanced cycle.

\section{Normalized Laplacian Matrix and Signed Network}

For a signed network $\Gamma$, the normalized Laplacian matrix $\mathscr{L}(\Gamma)$ is symmetric and positive semidefinite [7], so its eigenvalues are real and nonnegative, denoted by $0 \leq \lambda_{1} \leq$ $\lambda_{2} \leq \cdots \leq \lambda_{n}$. Firstly we recall some properties of normalized Laplacian matrix.

Lemma 11 (see [7]). Let $\Gamma$ be a signed network on $n$ vertices with normalized Laplacian eigenvalues $\lambda_{1} \leq \lambda_{2} \leq \cdots \leq \lambda_{n}$. Then $\lambda_{n} \leq 2$.

Lemma 12 (see $[3,7])$. Let $\Gamma_{1}$ and $\Gamma_{2}$ be two signed networks with the same underlying network. Then $\Gamma_{1} \sim \Gamma_{2}$ if and only if $\mathscr{L}\left(\Gamma_{1}\right)$ and $\mathscr{L}\left(\Gamma_{2}\right)$ are signature similar.

In [8], the symmetry about 1 of eigenvalues for bipartite signed network was present as follows. Here we present a stronger result. 
Theorem 13 (see [8]). Let $\Gamma=(G, \sigma)$ be a bipartite signed network. If $\lambda$ is an eigenvalue of $\mathscr{L}(\Gamma)$, then $2-\lambda$ is also an eigenvalue of $\mathscr{L}(\Gamma)$.

Theorem 14. Let $\Gamma$ be a connected signed network. Then $\Gamma$ is bipartite if and only if all eigenvalues of $\mathscr{L}(\Gamma)$ are symmetric about 1 (including multiplicities); i.e., for each eigenvalue $\lambda_{i}$, $2-\lambda_{i}$ is also an eigenvalue of $\mathscr{L}(\Gamma)$.

Proof. It suffices to verify that $I-\mathscr{L}(\Gamma)$ and $-(I-\mathscr{L}(\Gamma))$ have the same spectrum. Note that $I-\mathscr{L}(\Gamma)=D^{-1 / 2} A(\Gamma) D^{-1 / 2}$. $\Gamma$ is bipartite if and only if $D^{-1 / 2} A(\Gamma) D^{-1 / 2}$ can be expressed as $\left(\begin{array}{cc}0 & B \\ B^{T} & 0\end{array}\right)$. It is evident that

$$
\left(\begin{array}{cc}
-I & 0 \\
0 & I
\end{array}\right)\left(\begin{array}{cc}
0 & -B \\
-B^{T} & 0
\end{array}\right)\left(\begin{array}{cc}
-I & 0 \\
0 & I
\end{array}\right)=\left(\begin{array}{cc}
0 & B \\
B^{T} & 0
\end{array}\right)
$$

This yields to the result.

From Lemma 11, the integer 2 is the upper bound of normalized Laplacian eigenvalues. In this sequel, we give a sufficient and necessary condition for that the integer 2 is an eigenvalue of normalized Laplacian matrix.

Theorem 15. Let $\Gamma$ be a connected signed network. Then 2 is an eigenvalue of $\mathscr{L}(\Gamma)$ if and only if $\Gamma$ is a balanced bipartite signed network.

Proof. By Courant-Fischer theorem, we have

$$
\lambda_{n}=\sup _{f \neq 0} \frac{\sum_{u \sim v}(f(u)-\sigma(u v) f(v))^{2}}{\sum_{v} f^{2}(v) d(v)} .
$$

Assume that 2 is an eigenvalue of $\mathscr{L}(\Gamma)$ with nonzero eigenvector $y^{T}=\left(y_{1}, y_{2}, \ldots, y_{n}\right)$. By Lemma 11 and (14), $y_{i}=-\sigma\left(v_{i} v_{j}\right) y_{j}$ for any edge $e$ incident to $v_{i}$ and $v_{j}$. So $V(\Gamma)$ can be partitioned into two parts such that no edge existing between any two vertices in every part. This means that $\Gamma$ is bipartite. For any even cycle $C_{2 k}=v_{1} v_{2} \cdots v_{2 k} v_{1}$, we have

$$
\begin{aligned}
y_{1} & =-\sigma\left(v_{1} v_{2}\right) y_{2}=\sigma\left(v_{1} v_{2}\right) \sigma\left(v_{2} v_{3}\right) y_{3} \\
& =-\sigma\left(v_{1} v_{2}\right) \sigma\left(v_{2} v_{3}\right) \sigma\left(v_{3} v_{4}\right) y_{4}=\cdots \\
& =-\sigma\left(v_{1} v_{2}\right) \sigma\left(v_{2} v_{3}\right) \cdots \sigma\left(v_{2 k-1} v_{2 k}\right) y_{2 k} .
\end{aligned}
$$

Moreover, $y_{1}=-\sigma\left(v_{1} v_{2 k}\right) y_{2 k}$. So $\sigma\left(v_{1} v_{2}\right) \sigma\left(v_{2} v_{3}\right) \cdots \sigma\left(v_{2 k-1} v_{2 k}\right)=$ $\sigma\left(v_{1} v_{2 k}\right)$ and $C_{2 k}$ is balanced. This implies that $\Gamma$ is balanced.

If $\Gamma$ is balanced bipartite, then 0 is an eigenvalue of $\mathscr{L}(\Gamma)$. By Theorem 14 and Lemma 12, 2 is an eigenvalue of $\mathscr{L}(\Gamma)$.

As we know, the coefficients of characteristic polynomial of adjacency (Laplacian) matrix are related to the graph structure. In [9], expressions of coefficients of (Laplacian) characteristic polynomial was present. We would present the expression of the coefficients of normalized Laplacian characteristic polynomial. Firstly, we recall the Sachs formula for the coefficients of adjacency characteristic polynomial of signed networks. Here some definitions are needed. An elementary figure is the graph $K_{2}$ or the cycle. A basic figure is the disjoint union of elementary figures.
Lemma 16 (see [9]). Let $\Gamma=(G, \sigma)$ and $\phi(\Gamma, x)=x^{n}+a_{1} x^{n-1}+$ $\cdots+a_{n}$ be a signed network and its adjacency characteristic polynomial, respectively. Then

$$
a_{i}=\sum_{B \in \mathscr{B}_{i}}(-1)^{p(B)} 2^{|c(B)|} \sigma(B),
$$

where $\mathscr{B}_{i}$ is the set of basic figures on $i$ vertices in $G, p(B)$ is the number of components of $B$, and $c(B)$ is the set of cycles in $B$ and $\sigma(B)=\prod_{C \in c(B)} \operatorname{sgn}(C)$.

Let $\psi(\Gamma, x)$ be the normalized Laplacian characteristic polynomial of $\Gamma$. By the definition of normalized Laplacian matrix, we have

$$
\begin{aligned}
\psi(\Gamma, x)= & \operatorname{det}(x I-\mathscr{L}(\Gamma)) \\
= & \operatorname{det}\left(x I-D^{-1 / 2} L(\Gamma) D^{-1 / 2}\right) \\
= & \operatorname{det}\left(x I-I+D^{-1 / 2} A(\Gamma) D^{-1 / 2}\right) \\
= & \operatorname{det}\left((x-1) I+D^{-1 / 2} A(\Gamma) D^{-1 / 2}\right) \\
= & (x-1)^{n}+c_{1}(x-1)^{n-1}+\cdots+c_{n-1}(x-1) \\
& +c_{n} .
\end{aligned}
$$

Theorem 17. Let $\Gamma$ be a signed network on $n$ vertices and $\psi(\Gamma, x)=(x-1)^{n}+c_{1}(x-1)^{n-1}+\cdots+c_{n-1}(x-1)+c_{n}$ be its normalized Laplacian characteristic polynomial. Then

$$
c_{k}=\sum_{B \in \mathscr{B}_{k}}(-1)^{p(B)} 2^{|c(B)|} \sigma(B) \frac{1}{D_{k}},
$$

where $\mathscr{B}_{k}$ is the set of basic figures on $k$ vertices in $G, p(B)$ is the number of components of $B, c(B)$ is the set of cycles in $B$, $\sigma(B)=\prod_{C \in c(B)} \operatorname{sgn}(C), D_{k}=\prod_{v_{i} \in V(B)} d_{i}$, and $d_{i}$ is the degree of $v_{i}$ in $\Gamma$.

Proof. Note that

$$
\begin{aligned}
\psi(\Gamma, x) & =\operatorname{det}\left((x-1) I+D^{-1 / 2} A(\Gamma) D^{-1 / 2}\right) \\
& =(-1)^{n} \operatorname{det}\left((1-x) I-D^{-1 / 2} A(\Gamma) D^{-1 / 2}\right) .
\end{aligned}
$$

Set

$$
\begin{aligned}
\operatorname{det} & \left((1-x) I-D^{-1 / 2} A(\Gamma) D^{-1 / 2}\right) \\
= & (x-1)^{n}+c_{1}^{\prime}(x-1)^{n-1}+\cdots+c_{n-1}^{\prime}(x-1)+c_{n}^{\prime} .
\end{aligned}
$$

So $c_{k}=(-1)^{k} c_{k}^{\prime}$. Moreover, $(-1)^{k} c_{k}^{\prime}$ equals to the sum of all $k \times k$ minors of $D^{-1 / 2} A(\Gamma) D^{-1 / 2}$. Then $c_{k}$ is the sum of all $k \times k$ minors of $D^{-1 / 2} A(\Gamma) D^{-1 / 2}$. It is evident that each such $k \times k$ minor of $D^{-1 / 2} A(\Gamma) D^{-1 / 2}$ is the product of the corresponding $k \times k$ minors of $D^{-1 / 2}, A(\Gamma)$, and $D^{-1 / 2}$, respectively. Furthermore, any $k \times k$ minor of $A(\Gamma)$ is the determinant of adjacency matrix of an induced subgraph of $\Gamma$ with $k$ vertices. So this result holds from Lemma 16 . 


\section{Conclusion}

Recently, there are some results on the spectral theory of signed graphs [10-18]. In this paper we investigate some properties of (normalized) Laplacian matrix of signed network and present a correspondence between the balance of signed networks and the singularity of Laplacian matrix. Moreover, we give the expressions of determinant of Laplacian matrix and coefficients of normalized Laplacian characteristic polynomial, respectively. Actually there are some other aspects of spectrum of signed graphs, which can be investigated. It will be left to our future study. In addition, there are many spectrum-based invariants, which are widely investigated, such as graph energy (e.g., graph theory [19, 20], incidence energy [21], and matching energy [22, 23]), HOMO-LUMO index [24, 25], and inertia [26-29]. In the future, we would like to study some properties of these spectrum-based indices of signed networks.

\section{Data Availability}

No data were used to support this study.

\section{Conflicts of Interest}

The authors declare that they have no conflicts of interest.

\section{Acknowledgments}

This work was supported by the Natural Science Foundation of China (nos. 11301302, 11861019), the Natural Science Foundation of Shandong (no. BS2013SF009), and Foundation of Shandong Provincial Education Department (no. J17KA165).

\section{References}

[1] F. Heider, "Attitude and cognitive organization," The Journal of Psychology, vol. 21, pp. 107-122, 1946.

[2] D. Cartwright and F. Harary, "Structural balance: a generalization of Heider's theory," Psychological Review, vol. 63, no. 5, pp. 277-293, 1956.

[3] Y. Hou, J. Li, and Y. Pan, "On the Laplacian eigenvalues of signed graphs," Linear and Multilinear Algebra, vol. 51, no. 1, pp. 21-30, 2003.

[4] F. Harary, "On the notion of balance of a signed graph," Michigan Mathematical Journal, vol. 2, no. 2, pp. 143-146, 1953.

[5] G. Yu, X. Liu, and H. Qu, "Singularity of Hermitian (quasiLaplacian matrix of mixed graphs," Applied Mathematics and Computation, vol. 293, pp. 287-292, 2017.

[6] A. H. Roger and R. J. Charles, Matrix Analysis, Cambridge University Press, New York, NY, USA, 2nd edition, 2013.

[7] H.-H. Li and J.-S. Li, "Note on the normalized Laplacian eigenvalues of signed graphs," The Australasian Journal of Combinatorics, vol. 44, pp. 153-162, 2009.

[8] F. M. Atay and H. Tunçel, "On the spectrum of the normalized Laplacian for signed graphs: interlacing, contraction, and replication," Linear Algebra and Its Applications, vol. 442, pp. 165-177, 2014.
[9] F. Belardo and S. K. Simic', "On the Laplacian coefficients of signed graphs," Linear Algebra and Its Applications, vol. 475, pp. 94-113, 2015.

[10] Y.-Z. Fan, Y. Wang, and Y. Wang, "A note on the nullity of unicyclic signed graphs," Linear Algebra and Its Applications, vol. 438, no. 3, pp. 1193-1200, 2013.

[11] Y.-Z. Fan, W.-X. Du, and C.-L. Dong, "The nullity of bicyclic signed graphs," Linear and Multilinear Algebra, vol. 62, no. 2, pp. 242-251, 2014.

[12] I. Gutman, S.-L. Lee, J.-H. Sheu, and C. Li, "Predicting the, nodal properties of molecular orbitals by means of signed graphs," Bulletin of the Institute of Chemistry, Academia Sinica, vol. 42, pp. 25-32, 1995.

[13] Y. P. Hou, "Bounds for the least Laplacian eigenvalue of a signed graph," Acta Mathematica Sinica, vol. 21, no. 4, pp. 955-960, 2005.

[14] S. Lee and C. Li, "Chemical signed graph theory," International Journal of Quantum Chemistry, vol. 49, no. 5, pp. 639-648, 1994.

[15] S.-L. Lee, R. R. Lucchese, and S. Y. Chu, “Topological analysis of eigenvectors of the adjacency matrices in graph theory: the concept of internal connectivity," Chemical Physics Letters, vol. 137, no. 3, pp. 279-284, 1987.

[16] P. K. Sahu and S.-L. Lee, "Net-sign identity information index: A novel approach towards numerical characterization of chemical signed graph theory," Chemical Physics Letters, vol. 454, no. 1-3, pp. 133-138, 2008.

[17] Y. Liu and L. You, "Further results on the nullity of signed graphs," Journal of Applied Mathematics, vol. 2014, Article ID 483735, 8 pages, 2014.

[18] S. Wasserman and K. Faust, Social Networks Analysis: Methods And Applications, Cambridge University Press, Cambridge, 1994.

[19] R. Lang, T. Li, D. Mo, and Y. Shi, "A novel method for analyzing inverse problem of topological indices of graphs using competitive agglomeration," Applied Mathematics and Computation, vol. 291, pp. 115-121, 2016.

[20] X. Li, Y. Shi, and I. Gutman, Graph Theory, Springer, New York, NY, USA, 2012.

[21] S. B. Bozkurt and D. Bozkurt, "On incidence energy," MATCH Communications in Mathematical and in Computer Chemistry, vol. 72, no. 1, pp. 215-225, 2014.

[22] L. Chen, J. Liu, and Y. Shi, "Bounds on the matching energy of unicyclic odd-cycle graphs," MATCH - Communications in Mathematical and in Computer Chemistry, vol. 75, no. 2, pp. 315330, 2016.

[23] L. Chen, J. Liu, and Y. Shi, "Matching energy of unicyclic and bicyclic graphs with a given diameter," Complexity, vol. 21, no. 2, pp. 224-238, 2015.

[24] X. Li, Y. Li, Y. Shi, and I. Gutman, "Note on the HOMO-LUMO index of graphs," MATCH - Communications in Mathematical and in Computer Chemistry, vol. 70, no. 1, pp. 85-96, 2013.

[25] B. Mohar, "Median eigenvalues of bipartite planar graphs," MATCH - Communications in Mathematical and in Computer Chemistry, vol. 70, no. 1, pp. 79-84, 2013.

[26] G. Yu, H. Qu, and J. Tu, "Inertia of complex unit gain graphs," Applied Mathematics and Computation, vol. 265, pp. 619-629, 2015.

[27] G. Yu, L. Feng, and Q. Wang, "Bicyclic graphs with small positive index of inertia," Linear Algebra and its Applications, vol. 438, no. 5, pp. 2036-2045, 2013. 
[28] G. Yu, L. Feng, Q. Wang, and A. Ilic', “The minimal positive index of inertia of signed unicyclic graphs," Ars Combinatoria, vol. 117, pp. 245-255, 2014.

[29] G. Yu, X.-D. Zhang, and L. Feng, "The inertia of weighted unicyclic graphs," Linear Algebra and its Applications, vol. 448, pp. 130-152, 2014. 


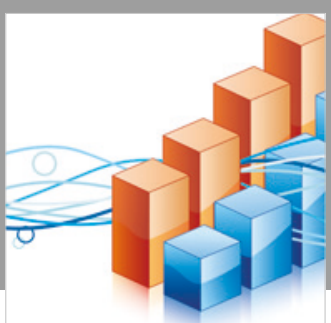

Advances in

Operations Research

\section{-n-m}
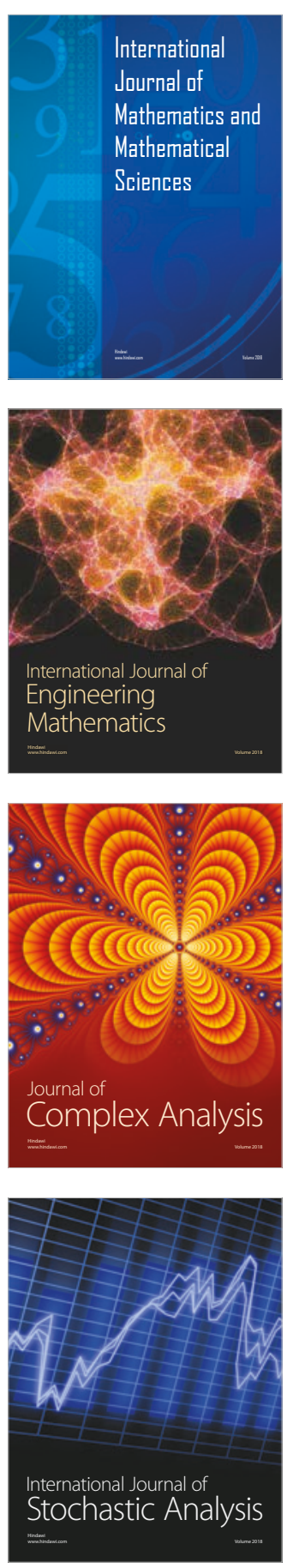
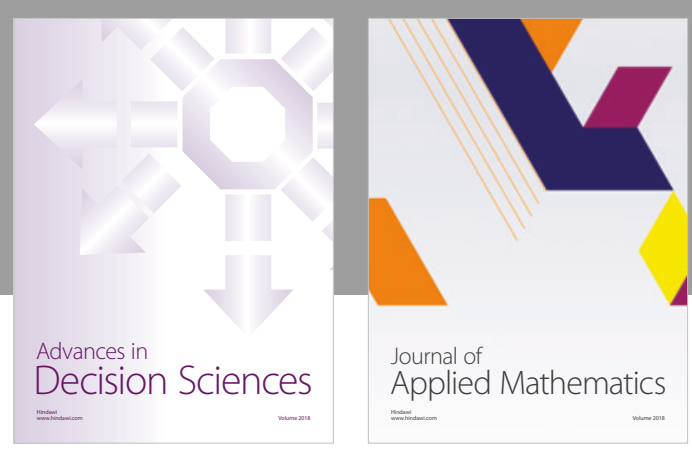

Journal of

Applied Mathematics
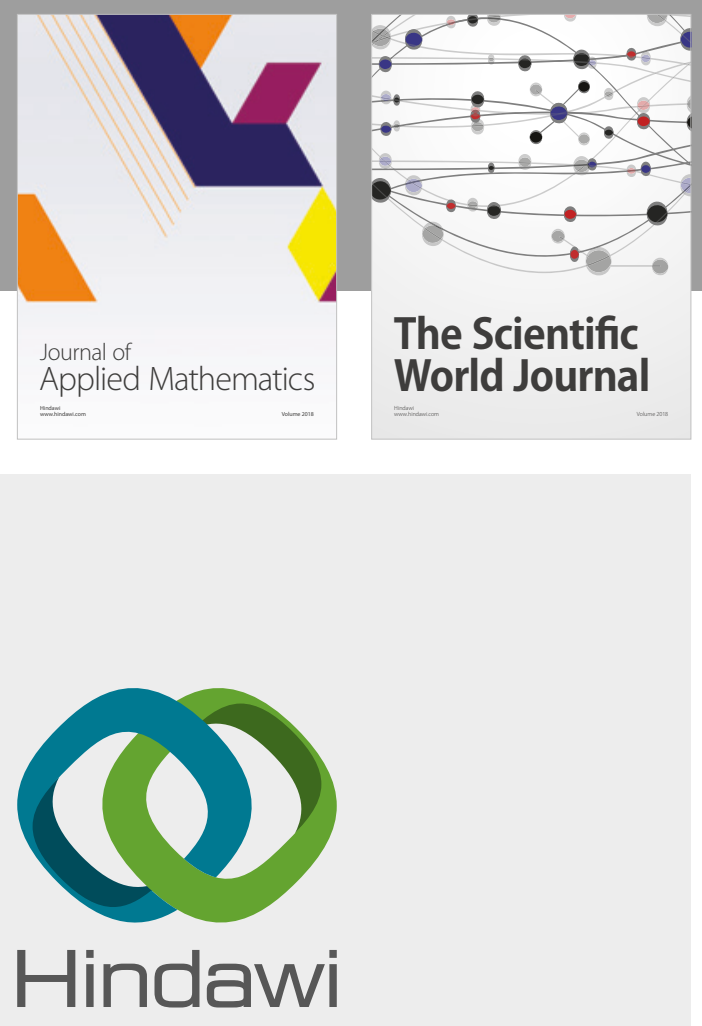

Submit your manuscripts at

www.hindawi.com

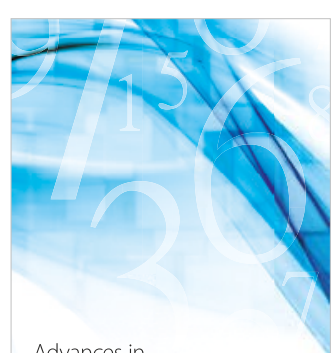

Advances in
Numerical Analysis
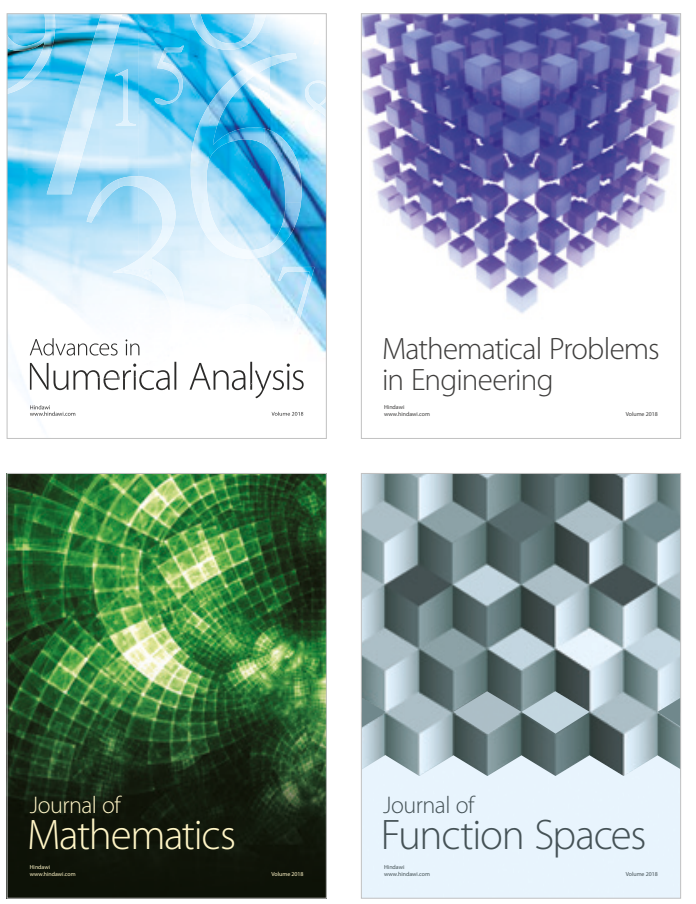

Mathematical Problems in Engineering

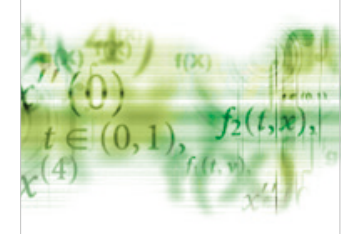

International Journal of

Differential Equations

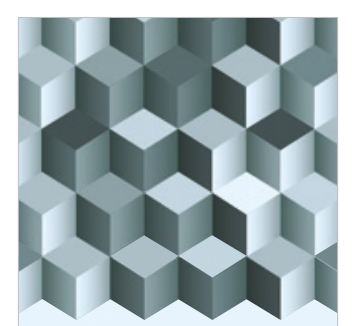

Journal of

Function Spaces
The Scientific

World Journal

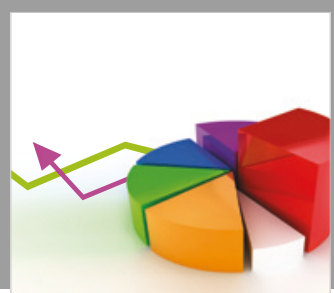

Journal of

Probability and Statistics
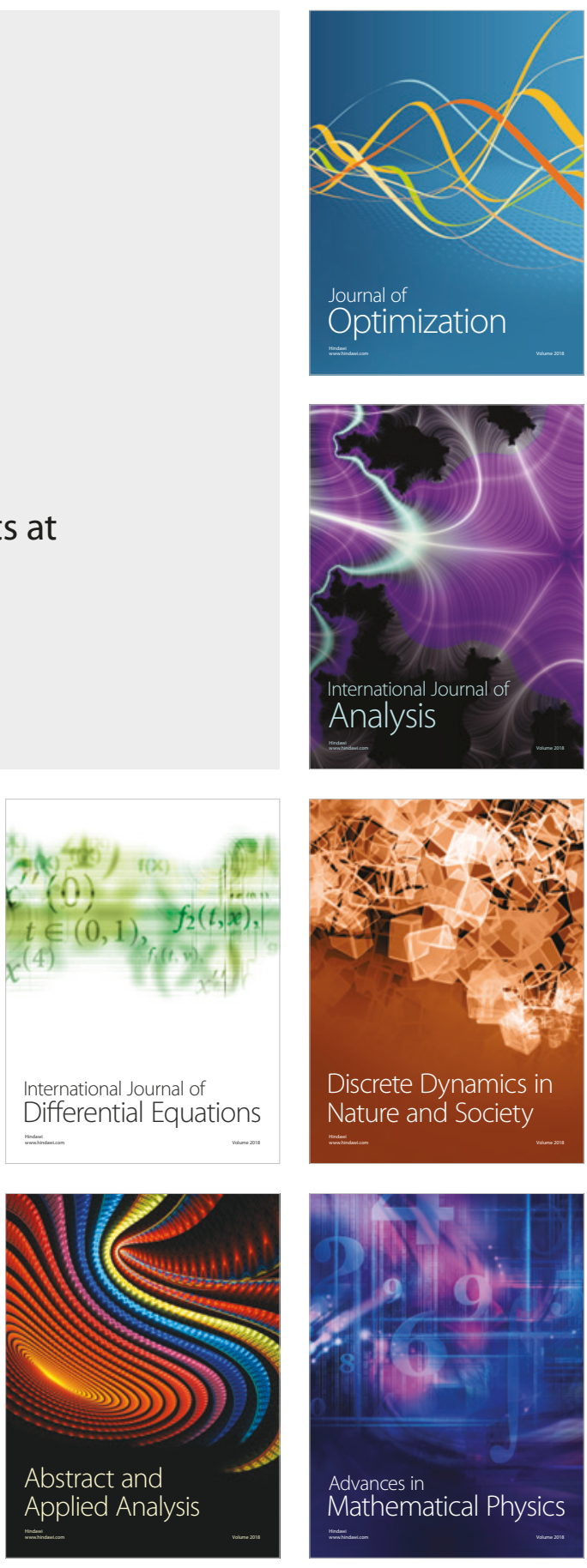